\title{
Design and Optimization of Indoor Space Layout Based on Deep Learning
}

\author{
Yingfei Sun \\ School of Art and Design, Geely University of China, Chengdu 641423, Sichuan, China \\ Correspondence should be addressed to Yingfei Sun; scisunyingfei@163.com
}

Received 30 December 2021; Revised 18 January 2022; Accepted 26 January 2022; Published 23 February 2022

Academic Editor: Hasan Ali Khattak

Copyright (@ 2022 Yingfei Sun. This is an open access article distributed under the Creative Commons Attribution License, which permits unrestricted use, distribution, and reproduction in any medium, provided the original work is properly cited.

\begin{abstract}
In order to explore the technical path of using artificial intelligence deep learning algorithm in realizing the interior space layout, this study introduces neural network modules such as 3D spatial convolutional (3DSC) neural networks and fuzzy neural networks (FNN), and a deep learning algorithm of indoor spatial layout design (ISLD) based on the adversarial neural network (ANN) is formed. In the algorithm design, a controllable data-interference adverse variation algorithm based on a random number generator is introduced, to obtain the data variant optimization process of genetic algorithm in neural network deep learning. As shown in the simulation analysis, the algorithm yielded significantly better subjective audience evaluation than other algorithms mentioned in references, and because it can be run offline on a single PC workstation, the demand for network resources and computing power resources is relatively small, so under the premise of the same hardware facility investment, higher production capacity can be obtained to get a higher input-output ratio, and it has a certain industry-university-research transformation and market promotion value.
\end{abstract}

\section{Introduction}

Layout design refers to the combination of dividing a given space into some small spaces or reasonably arranging several objects to be arranged in the space, meeting the constraints of some objective and subjective layout conventions and design criteria, and finally making the layout meet the customer's satisfaction through optimization and adjustment [1]. Because home decoration design belongs to the category of engineering art design, the participation of artificial intelligence algorithm in art design has always been the biggest difficulty. Relevant research found that although the deep learning function of artificial intelligence algorithm cannot participate in the whole process of home decoration design, it has certain usability in spatial layout design.

Indoor space layout includes the design of layout plan (i.e., space division) and layout optimization of indoor scene (i.e., home layout) [2]. Interior space layout is an important part of interior decoration. People's living environment can be more comfortable through interior space design. With the improvement of people's material living standards, there are more and more requirements for indoor space layout design [3]. The traditional interior space layout design is designed manually by the designer, which is limited by the designer's professional ability and knowledge level. In the traditional layout design, a lot of professional knowledge, creative intuition, and design experience are required, and the design process will consume a lot of energy and manpower [4]. With the development of computer technology, there are more and more modeling software using the computeraided interior layout. Designers use interactive modeling software to assist design. Relevant software for layout design includes software that provides solutions for professionals, such as ArchiCAD, Vectorworks, and general software focusing on indoor scene layout, such as Roomarranger, Live Home 3D. Some mainstream 3d modeling software also includes the design function of spatial layout, such as 3dsmax, AutoCAD. [5]. However, this software has certain limitations because they require a large number of user interactions, high learning threshold, low degree of automation and intelligence, simple and immature existing indoor space layout design rules, unsatisfactory design effect, 
and other problems and are not suitable for large-scale application.

By changing the traditional design model, the automatic design of the spatial layout is a research problem to solve in contemporary academia. This study introduces deep learning-based intelligent design methods in interior spatial layout design, designs an interior space layout design tool, simulates designer ideas during the design process, realizes automation or intelligent design, effectively solves the problem of time-consuming and power-consuming manual layout, provides the best design solution, and improves the overall level of the field of interior design. The application of intelligent design also discusses the application prospect of the design and optimization of artificial intelligence technology in the indoor spatial layout.

\section{Interior Space Layout Countermeasure Neural Network Design Based on Deep Learning}

At this stage, with the gradual maturity and rapid popularization of artificial intelligence, the research on layout design is also increasing. Han et al. studied a layout design system with constraint combinations, which can generate a set of configurations based on a given constraint, and it is also prioritized according to multiple evaluation indicators to find the best functional configuration for the rooms in the layout. However, because of the complexity of the combination problem, the efficiency is relatively low [6]. Bahrehmand et al. studied the interactive layout design system using an evolutionary algorithm, which can generate personalized spatial layout according to customer preferences and design criteria, but the optimization effect is not satisfactory [7].

Interior space layout is a special constraint satisfaction problem (CSP), which can select the optimal result in the fixed layout scheme. Combinatorial optimization is the optimal programming of discrete problems based on mathematical models [8].

We regard the interior space layout problem as a general combinatorial optimization problem, define its feasible solution set as the feasible region $\mathrm{F}$, and obtain the following formula:

$$
F=\{x \mid x \in D, g(x) \geq 0\},
$$

where $x$ is configuration variables in indoor space layout; $D$ is definition domain of configuration variable; $g(x)$ is constraint equation; $F$ contains finite feasible solutions. In order to obtain the optimal solution, we only need to judge the feasible solutions in the feasible region one by one and compare the size of the objective function value $f(x)$. However, there are many feasible solutions, which need to be optimized with the help of neural network and deep learning.

The application of deep neural network, especially convolution neural network, has brought a new research direction for the design of indoor space layout [9]. Neural network simulates the function of human brain neurons and has strong data processing ability and simulated learning ability. Fuzzy logic method simulates the logical thinking mode of the human brain and implements fuzzy comprehensive judgment and reasoning to solve the structural knowledge expression ability that is difficult to deal with by conventional methods [10]. Fuzzy neural grid combines the structure of the human brain with thinking methods, learns from each other, overcomes the poor processing of fuzzy data at the boundary of the traditional neural network, and carries out learning and adjustment [11]. Fuzzy neural network algorithm can establish the hidden logical relationship between the statistical data and the control strategy according to the hiding law of incomplete data [12]. The minimum mode of fuzzy neural network is shown in Figure 1.

The system collects data first. After deep neural network learning, the customer inputs the specified conditions to generate the qualified indoor space layout design drawing. The system plans to use the deep learning countermeasure neural network composed of spatial segmentation generation algorithm and verification algorithm as the core algorithm. The flow chart of interior space layout design based on a deep neural network system is shown in Figure 2.

We collected a large number of CAD indoor layout plans as data sets, entered the three-dimensional space convolution model, trained the logarithmic depth iterative convolution model through the polynomial regression model, and generated the selected indoor space layout design according to the conditions specified by the customer. After the CAD original spatial model is imported, the three-dimensional spatial convolution method is used for data preprocessing. The processed data are convoluted into a double-precision floating-point variable using fuzzy neural network (FNN) as the reference data. The data are divided into two ways. One way is input into the threedimensional space generation module as the convolution kernel parameter to convolute the original CAD drawing data to form the space division result, and the other way is input into the antineural network evaluation module (GAN). When the evaluation result is greater than 0.950, the data are considered qualified, and the three-dimensional space generation algorithm is driven to output the final data. When the evaluation result is less than 0.950 , the data are considered unqualified, and the recalculation trigger process is driven. The algorithm mainly has the following three technical points.

\subsection{Data Mutation Evolution under Random Number} Interference. In this paper, the FNN algorithm is used to form the preliminary spatial convolution results into reference data. During the data generation process, the interference data on the $[0,1]$ interval generated by the random number generator are imported, and the reference data are returned to the three-dimensional space generation module for secondary spatial convolution. During secondary spatial convolution, the original CAD threedimensional model data are reread. The original CAD 3D model data are the original measurement data of the designed space. 


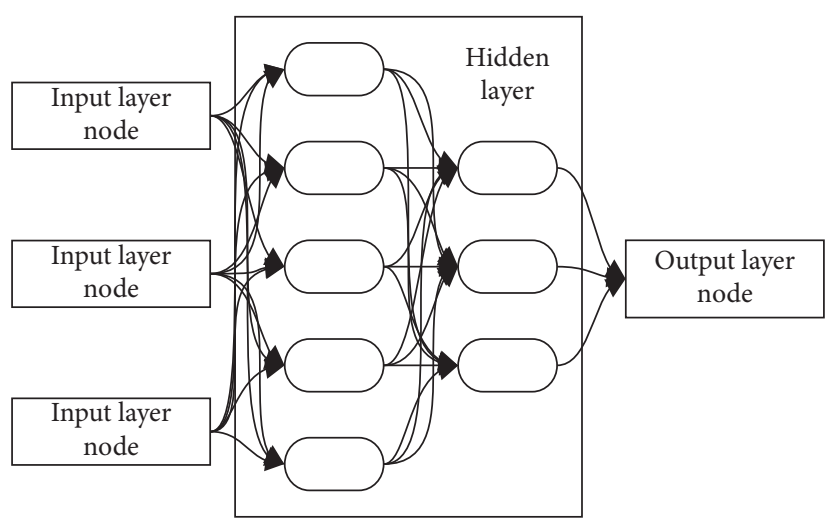

Figure 1: Minimum mode diagram of the fuzzy neural network.

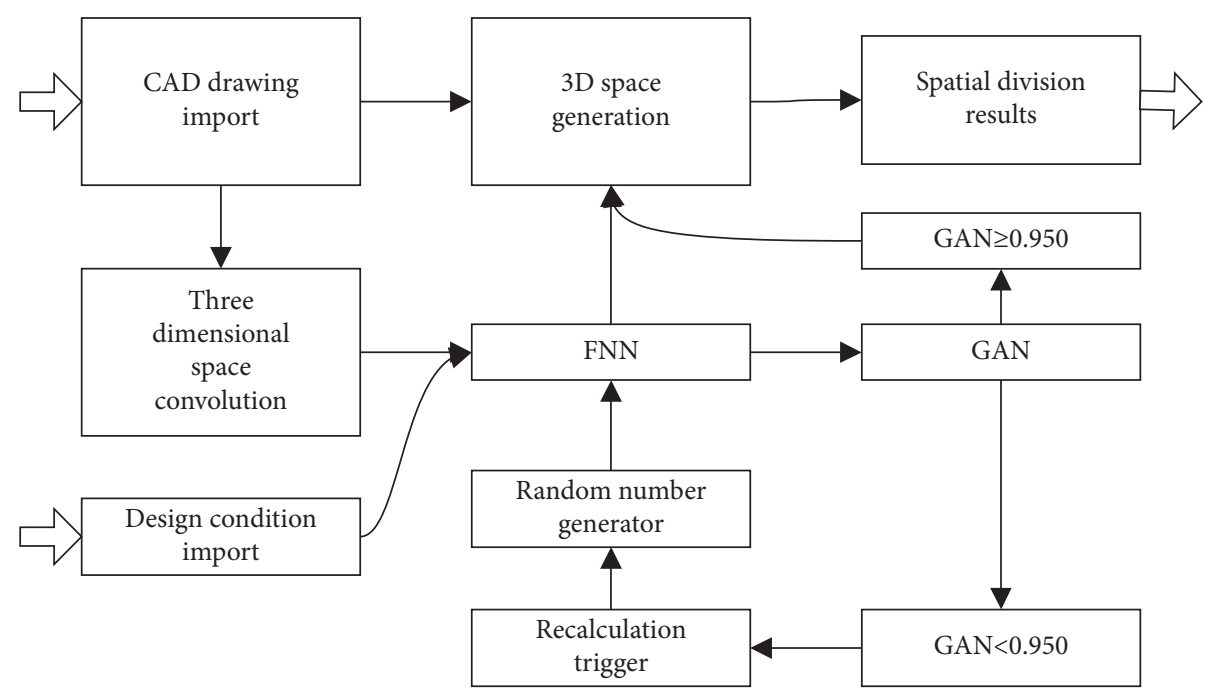

FIGURE 2: Schematic diagram of layout design flow based on the spatial convolution neural network.

2.2. Low Intervention Learning of Left-Right Competition in FNN-GAN Confrontation. The reference data output by the FNN algorithm module is used as the analysis basis of the GAN algorithm module, rather than inputting the final output results into the GAN algorithm module for analysis. This technology is the innovation of the research and design algorithm. The algorithm innovation helps to reduce the computational power demand of the GAN algorithm, improve the computational efficiency, and reduce the hardware demand. The system can run on a PC workstation system without building an expensive big data analysis host system.

2.3. The Final Spatial Model Division Result Formed by Twice Spatial Convolution Combined with FNN. The spatial convolution process of this study is divided into two steps: the first step is to directly convolute the original data based on edge enhancement and data association to form a threedimensional model of structural data enhancement, which forms a reference value after FNN module fuzzy convolution analysis with strong interference. The second step is to read the original data again and form the convolution result of the final three-dimensional design model driven by the reliable reference value output by the FNN module.

\section{Detailed Design of the Indoor Space Layout System Based on Deep Learning}

CAD drawings and customer design conditions are variables. These two variables need to be converted into floatingpoint variables (double type) after forced normalization. The normalization method is Z-score algorithm, and its basis function is shown in the following formula:

$$
\begin{aligned}
Z_{i} & =\frac{X_{i}-\bar{X}}{\sigma}, \\
\sigma & =\frac{1}{n-1} \sqrt{\sum_{i=1}^{n}\left(X_{i}-\bar{X}\right)^{2}}, \\
\bar{X} & =\frac{1}{n} \sum_{i=1}^{n} X_{i} .
\end{aligned}
$$


Here, $\sigma$ is the standard deviation rate of sequence $x ; \bar{X}$ is the arithmetic mean of sequence $x ; n$ is the maximum subscript of sequence $x$, that is, the number of elements it contains; $X_{i}$ is the $i$ th element value used for calculation in sequence $x$; $Z_{i}$ is the normalized output value corresponding to $X_{i}$.

In order to realize three-dimensional space convolution and three-dimensional space generation, it is suitable for three-dimensional data superposition neural network basis function, and its node function is as shown in the following formula:

$$
y=\int_{-\infty}^{+\infty} g(x) j(a-x) k(a-x) q(a-x) \mathrm{d} x,
$$

where $g(x)$ is convolution kernel; $j(A-X), k(A-X)$ and $q(A-X)$ are three-dimensional original images, respectively; $x$ is the traversal variable; $a$ is the auxiliary positioning variable; $y$ is the output value of neural network. In order to use the minimum number of nodes to realize the fuzzy convolution of data, it is necessary to use the sixth-order polynomial depth iterative regression basis function (FNN) with complex function curve and rich regression coefficients. Its expression is shown in the following formula:

$$
y=\sum_{i=1}^{n} \sum_{j=0}^{5} A_{j} x_{i}^{j}
$$

where $A_{j}$ is coefficients to be regressed of the $j$-th order polynomial; that is, each node in the formula contains 6 coefficients to be regressed from $A_{0}$ to $A_{5} ; j$ is polynomial order. The meanings of other mathematical symbols are the same as those above.

In most image data, there are local dense distribution and long-distance outlier data, so a variety of nonlinear functions need to be used for data statistical analysis in neural network analysis. For example, the log depth iterative analysis method will shift the data close to the 0 value of the number axis to the right end of the number axis, making the data distribution more average, but does not change the relationship between the data landing points. The basis function of the logarithm depth iterative regression neural network (GAN) module is shown in the following formula:

$$
y=\sum_{i=1}^{n}\left(A \cdot \log x_{i}+B\right)
$$

where $A$ and $B$ are coefficients to be regressed. The meanings of other mathematical symbols are the same as those above.

\section{Correlation Analysis between Computational Power Input and Subjective Satisfaction of Deep Learning Neural Network}

The simulation software of the above algorithm is constructed in MATLAB, and the spatial division result data that have previously obtained high customer subjective satisfaction are used as the training data to fully converge the deep learning module. In order to achieve a more efficient machine learning process, the comparison relationship between neural network node investment and customer subjective satisfaction is discussed. The observation results are shown in Table 1.

As shown in Table 1, number of generation module nodes: the number of total neural network nodes in the generation module, unit $\mathrm{K}$; Number of evaluation module nodes: the number of total neural network nodes in the evaluation module, unit $\mathrm{K}$; The data in the table is the average value of users' subjective evaluation results, with a full score of 10 and a minimum score of 0 ; This table statistics the relationship between the computing power input and the subjective customer satisfaction. The data in the table are the average value of users' subjective evaluation results, with a full score of 10 and a minimum score of 0 . In order to facilitate the observation of the data in the table, the data visualization is performed on the data in the table, and Figure 3 is obtained.

In Figure 3, the legend data represent the number of evaluation module nodes $(k)$. The meaning of other data is the same as that in Table 1. As can be seen from the data in Figure 3, increasing the number of generation module nodes will significantly improve the user's satisfaction with the division of artificial intelligence deep learning space. Especially, when the number of generation module nodes reaches more than $45 \mathrm{k}$ nodes, the customer's subjective evaluation results converge to more than 9.0 points, and the increase slows down. It can be concluded that when the optimization of the number of generation module nodes reaches $45 \mathrm{k}$ nodes, the system reaches the optimal value under the constraint of economic indicators. Adding the number of evaluation module nodes has no significant impact on the actual output of the system and customer subjective satisfaction; that is, the optimization process of the system for the number of evaluation module nodes only needs to minimize the number of nodes required for system operation.

\section{Comparison between the Software and Similar Software}

Similarly, the simulation software supported by the algorithm in the Matlab environment is built, the simulation software supported by the algorithm given in other relevant literature is reproduced and built, and the actual effects of different algorithms are compared. The comparison algorithms include the strong stem prespace convolution depth learning algorithm (hereinafter referred to as algorithm A), the depth learning algorithm based on cellular automata (hereinafter referred to as algorithm B), and the depth learning algorithm based on ant colony genetic algorithm (hereinafter referred to as algorithm $\mathrm{C}$ ), and the above algorithms are leading in the relevant literature. Because all algorithms have not formed commercial software, all algorithm comparison processes are executed in the simulation environment.

5.1. Comparison of Users' Subjective Satisfaction. For 500 commercial housing plans, the original CAD housing structure drawings are constructed, and the above four algorithms are used for space division, including the demolition and reconstruction of structural walls, the replanning of door and window positions, the division of jump floor 
TABLE 1: Correlation statistics between computing power input and customer subjective satisfaction.

\begin{tabular}{lcccc}
\hline \multirow{2}{*}{ Number of generation module nodes $(k)$} & \multicolumn{4}{c}{ mumber of evaluation } \\
& 30 & 35 & 40 & 45 \\
\hline 30 & 6.12 & 6.35 & 6.42 & 6.44 \\
35 & 7.56 & 7.61 & 7.64 & 7.67 \\
40 & 8.53 & 8.64 & 8.67 & 8.69 \\
45 & 8.95 & 9.01 & 9.02 & 9.05 \\
50 & 9.08 & 9.12 & 9.14 & 9.16 \\
55 & 9.12 & 9.18 & 9.19 & 9.21 \\
60 & 9.15 & 9.22 & 9.25 & 9.28 \\
\hline
\end{tabular}

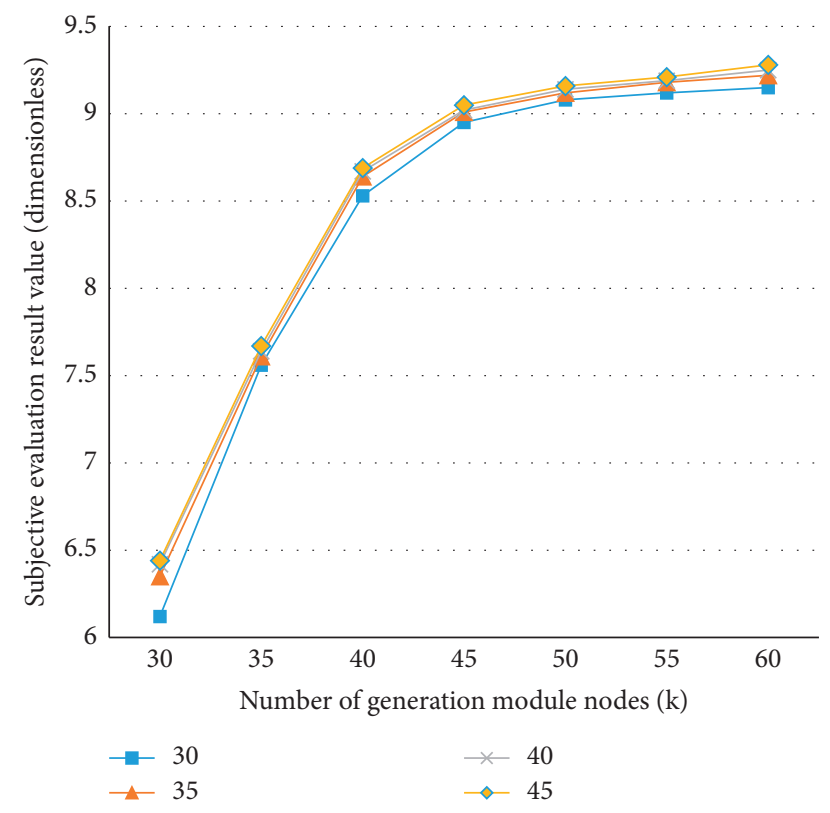

FIgURE 3: Statistical diagram of the correlation between computing power input and customer subjective satisfaction.

space, moving line planning. 2000 ordinary residents who have no practical experience in building decoration are invited as subjective evaluators, and they are required to give subjective evaluations on the degree of beauty, practicability, cost control ability, and comprehensive feeling, respectively. The highest evaluation result is 10 points, and the lowest is 0 points, and their arithmetic average value is calculated. The statistical results are shown in Table 2.

In Table 2, beautiful refers to users' subjective aesthetic evaluation of the final space division results; practical refers to user's subjective evaluation of the practicability of the final space division result; cost refers to the user's subjective evaluation of the cost control ability of the final space division result; and comprehensive refers to the overall evaluation result of the user on the final space division result.

After visual analysis of the data in Table 2, Figure 4 is obtained.

In Table 2 and Figure 4, although in all evaluation results, the lowest is 7.289 (algorithm B of cost comparison results), and the highest is 9.825 (the algorithm of cost comparison
TABLE 2: Comparison results of users' subjective satisfaction.

\begin{tabular}{lcccc}
\hline Algorithm & Beautiful & Practical & Cost & Comprehensive \\
\hline This algorithm & 9.303 & 9.132 & 9.825 & 9.245 \\
Algorithm A & 8.216 & 8.334 & 8.046 & 8.173 \\
Algorithm B & 8.986 & 9.273 & 7.289 & 8.926 \\
Algorithm C & 9.252 & 8.006 & 8.239 & 8.185 \\
\hline
\end{tabular}

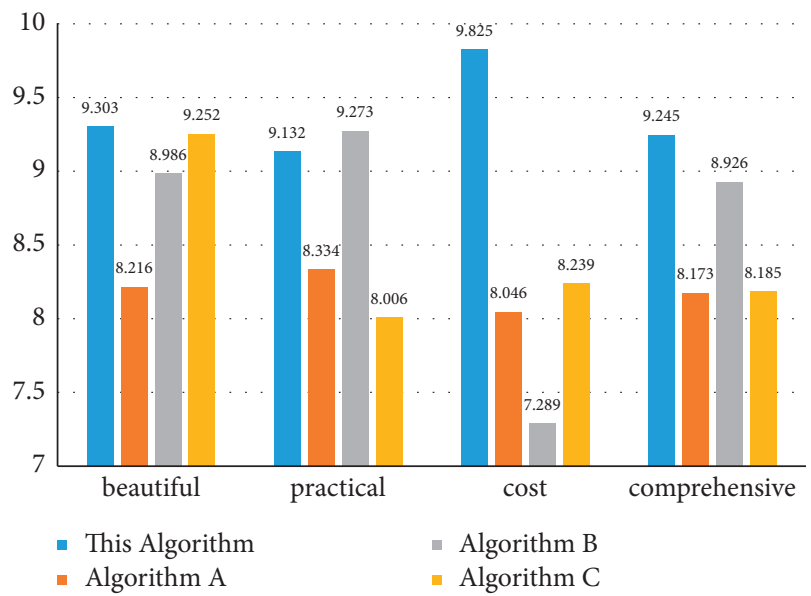

Figure 4: Comparison results of user subjective satisfaction.

results), the gap between the evaluation results is small, but there is still a certain data law in comparing the evaluation values of various algorithms, and among the evaluation values of the algorithm, the evaluation value of the practical comparison result is lower than that of algorithm $\mathrm{B}$, and the evaluation value of the algorithm is the highest among other evaluations. Based on a strong intervention spatial convolutional deep learning algorithm, the evaluation result of algorithm A is low and lacks leadership compared with the other three algorithms: deep learning algorithm based on cellular automata (algorithm B) is an alternative algorithm second only to the algorithm. Except that some evaluation values are slightly lower than the algorithm, it gives higher evaluation values in various evaluations, but its performance is poor in the cost comparison project. It can be considered that algorithm $B$ focuses on better design effect but has poor cost control ability; deep learning algorithm based on ant colony genetic algorithm (algorithm c) scored higher in aesthetic evaluation, second only to the algorithm, but other evaluation results were slightly worse.

5.2. Comparison of Input-Output Ratio. Because different algorithms occupy computing power equipment to different degrees, the algorithm can run on a single PC workstation. The minimum operation standard is 24 core $\mathrm{CPU}, 48$ core GPU, 32 GB central dynamic memory, and 128 GB floatingpoint dynamic memory. Other algorithms need to build computing center equipment supported by the mediumsized computer system. Although the final response time of the software system supported by the algorithm is long and the output efficiency is low, referring to the hardware input 
TABle 3: Comparison results of input-output ratio.

\begin{tabular}{lcc}
\hline Algorithm & $\begin{array}{c}\text { Unit capacity } \\
\text { Pieces per day }\end{array}$ & $\begin{array}{c}\text { Equivalent capacity } \\
\text { Pieces per M RMB }\end{array}$ \\
\hline This algorithm & 13.7 & 689.3 \\
Algorithm A & 26.5 & 126.9 \\
Algorithm B & 48.4 & 92.4 \\
Algorithm C & 22.6 & 205.8 \\
\hline
\end{tabular}

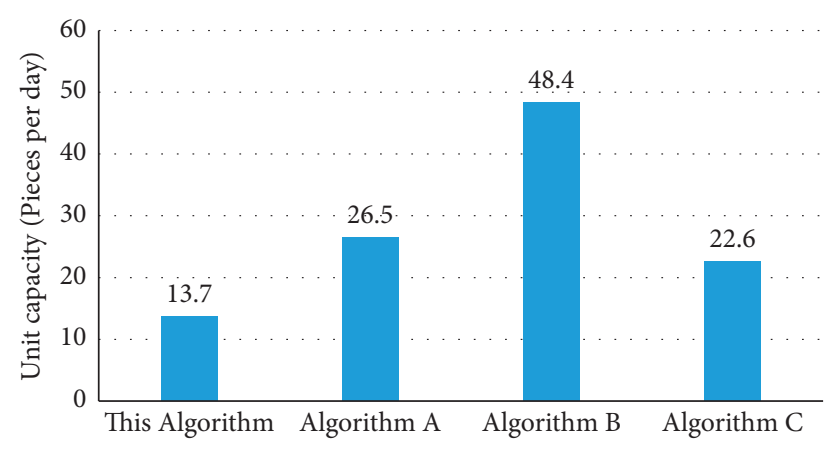

FIGURE 5: Unit capacity.

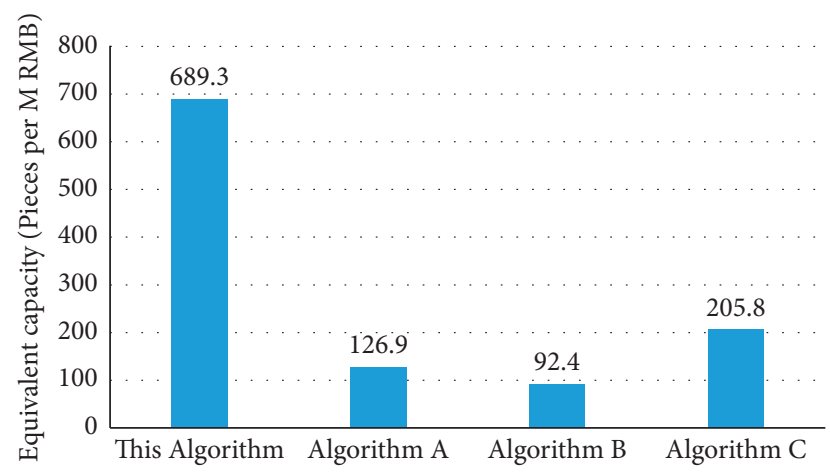

FIgURE 6: Equivalent capacity.

conditions, the input-output ratio of the algorithm-supported system should be higher than that of other systems. Specific comparison is made in the simulation environment, and Table 3 is obtained.

In Table 3, unit capacity refers to the number of space partitions that can be completed by each independent device every 24 hours (pieces per day); equivalent capacity refers to the number of space division pieces that can be completed every 24 hours for the overall equipment hardware invested and constructed with RMB 1 million; each of the above calculation tasks is $80-300$ square meters of commercial residential buildings, and the residential jump is $2.8-4.5$ meters, and the division requirements are within the scope of engineering realization. The operation tasks of all algorithms are completely consistent.

In order to fully observe the above data, visual analysis is performed on the data in Table 3 to obtain Figures 5 and 6 .

In Figure 5, the algorithm supported by the algorithm has a low daily output of a single device because of its low computing power. The daily output of a single device based on the deep learning algorithm and cellular automata (algorithm $\mathrm{B}$ ) is high, but the investment amount of a single device of algorithm $B$ is also large.

Under the same equipment hardware input conditions, the capacity data to obtain Figure 6 is recounted.

In Figure 6, because the investment of a single device required by the algorithm is less than RMB 20000 and RMB 1 million, the running hardware of the algorithm can deploy more than 50 devices. Other algorithms also arrange the equipment cluster according to the market price of RMB 1 million. Comparing the capacity under the same hardware investment conditions, it is found that the capacity of the algorithm software is much higher than that of other algorithms. Based on previous analysis, the audience evaluation single value and comprehensive value of the algorithm are high, and this is enough to prove the leadership of the algorithm.

\section{Summary}

With the improvement of living conditions, people have higher and higher requirements for indoor layout, but the previous indoor layout design is subject to various limitations, and the design process will consume a lot of energy and manpower. Then, the mature development of artificial intelligence makes people gradually increase the application and research of space layout design, but there are some problems, such as low efficiency and poor optimization effect. Based on previous studies, this study comprehensively applies spatial convolution neural network and fuzzy convolution neural network to form a weak intervention deep learning countermeasure neural network algorithm and realizes the space division algorithm in intelligent commercial housing decoration design based on machine learning. In the commercial housing decoration design, the algorithm enables the robot to independently complete the design tasks such as the demolition and reconstruction of structural walls, the replanning of door and window positions, the division of floor hopping space, and moving line planning and has achieved the results of artificial intelligence algorithm with high subjective evaluation results by the audience. However, the algorithm has not yet involved space art design, home decoration soft decoration design, home decoration art design, decoration construction design, and other links. In future research, for the field of deep learning involved in decoration design, we should also expand the application scenarios and gradually realize the full process of artificial intelligence deep learning design function.

\section{Data Availability}

All the data in this paper are from the raw data and the experimental results data collected during the experiment.

\section{Conflicts of Interest}

The author declares that there are no conflicts of interest. 


\section{Authors' Contributions}

The author has seen the manuscript and approved it for submission.

\section{References}

[1] H. Yuan, Z. Chen, and N. Sun, "Study on the spatial layout design of the sports and sports center," Industrial Design, vol. 33, no. 11, pp. 103-104, 2021.

[2] G. Y. Ping, "Application of humanized interior design in modern interior design.- takes the reasonable layout of decoration interior space as an example," Usher, vol. 93, no. 31, pp. 28-30, 2021.

[3] H. Zhong and X. Zheng, "Application of minimalism in modern home space," City Housing, vol. 28, no. 7, pp. 185-186, 2021.

[4] S. Yang, "Design of 3 D simulation imaging system for indoor space layout based on VR technology," Modern Electronics technology, vol. 44, no. 8, pp. 109-113, 2021.

[5] Y. Qin, "Computer-aided design in interior design performance," Furniture \& interior decor, vol. 36, no. 12, pp. 60-61, 2009.

[6] L. Han, L. Zhou, and J. Guo, "Automatic generation algorithm for indoor space topology model based on building plan," Mapping Bulletin, vol. 2021, no. 3, pp. 75-80+86, 2021.

[7] A. Bahrehmand, T. Batard, R. Marques, A. Evans, and J. Blat, "Optimizing layout using spatial quality metrics and user preferences," Graphical Models, vol. 93, 2017.

[8] W. Shuyan and Y. Zhang, "Research on interior design and transformation of self-built houses under new rural construction," Usher, vol. 96, no. 32, pp. 34-36, 2021.

[9] Q. Wang, "Practical study on the combination of interior design and intelligent home furnunder computer information technology," Computer Knowledge and Technology, vol. 17, no. 32, pp. 167-168+173, 2021.

[10] E. Karan, S. Asgari, and A. Rashidi, "A markov decision process workflow for automating interior design," KSCE Journal of Civil Engineering, vol. 25, no. 5, pp. 36-43, 2021.

[11] Qi. Zhang, "Aesthetic differences between Chinese and western cultures based on big data analysis," Journal of Physics: Conference Series, vol. 1852, no. 2, pp. 124-133, 2021.

[12] Y. Li, "Design analysis of white space technique in interior space," Journal of Design, vol. 05, no. 1, pp. 812-823, 2020. 